\title{
THE DISTORTION OF THE BOUSSINESQ FIELD DUE TO A CIRCULAR HOLE*
}

\author{
BY \\ A. BARJANSKY' \\ The Brush Development Company
}

1. Introduction. One of the most important problems in the theory of elasticity is the solution of the biharmonic equation $\nabla^{2} \phi=0$, where $\phi$ is Airy's stress function, for a given group of boundary conditions. As is well known, the most common approach to the solution of this problem consists in selecting a system of coordinates particularly suited to the region studied.

Thus, using bipolar coordinates, G. B. Jeffery has given the general solution of the plane problem, that is, of the biharmonic equation in two dimensions, for regions bounded by non-concentric circles (Ref. 1). A clear, but not quite complete, treatment of Jeffery's method can be found in Coker and Filon (Ref. 2). This method has recently been used by R. D. Mindlin for the determination of dead loads on tunnels (Ref. 3).

The present paper is an attempt to apply Jeffery's approach to the problem of the distortion introduced in the so-called plane Boussinesq field by the presence of a circular hole. Starting with the stress function $\phi$ of the undistorted Boussinesq field, an auxiliary stress function $\chi$ will be found such that $\Phi=\phi+\chi$ satisfies the differential equation and all the boundary conditions. The stresses and strains in the discontinuous field can then be directly determined from the derivatives of $\Phi$.

2. The Boussinesq field. Boussinesq and Flamant have given the solution of the biharmonic equation for the case of an isolated force $P$ acting at a point on the boundary of a semi-infinite plane. Their solution, which can be found in all standard texts (see, for instance, Ref. 4 , p. 82) is:

$$
\phi_{1}=-\frac{P}{\pi} r \theta \sin \theta
$$

for the case of a normal force, and

$$
\phi_{2}=-\frac{P}{\pi} r \theta \cos \theta
$$

for a force parallel to the boundary. The significance of the symbols is shown in Figs. $1 \mathrm{a}$ and $1 \mathrm{~b}$.

In the simple Boussinesq problem, the only boundary conditions are that the stresses, both normal and shearing, must vanish along the straight boundary (except, of course, at the point of application of the force) and also must tend to zero as one

* Received Sept. 23, 1943.

1 The writer wishes to express his thanks to the following persons who have assisted him in the preparation of this paper: Professor M. S. Ketchum of Case School of Applied Science, who suggested the problem; Dr. W. N. Dudley, also of Case, for several helpful hints; and especially Dr. H. G. Baerwald whose assistance in many mathematical details has been invaluable. 
moves away from the point of application within the half-plane. With the appearance of the circular discontinuity (Fig. 2), the above conditions remain, and a new one is

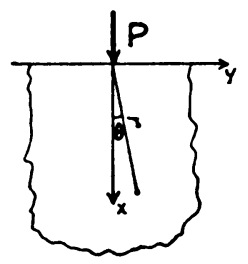

Fig. 1a.

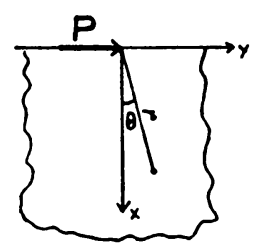

Fig. 1b.

added, determined by the nature of the discontinuity. Thus, if it is a hole, both normal and shearing stresses must vanish along its periphery.

The region is thus bounded by a circle and a straight line; the latter can be considered as a circle of infinite radius, so that here is a case of a region bounded by two non-concentric circles, to which Jeffery's method is applicable.

3. Bipolar coordinates. Jeffery's method consists essentially in introducing a system of curvilinear coordinates, called bipolar coordinates in works on elasticity. Two poles, $A$ and $B$ (Fig. 3) are taken at abscissas $\pm a$ along the $X$-axis, and the location of

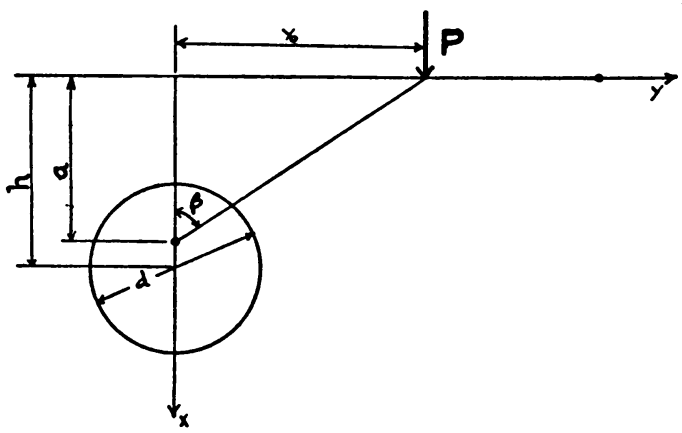

Fig. 2. any point is determined with respect to these poles by the quantities

$$
\xi=\log \frac{r_{1}}{r_{2}} \quad \eta=\theta_{1}-\theta_{2} .
$$

The lines $\eta=$ constant are circles passing through $A$ and $\dot{B}$, while $\xi=$ constant are a system of circles with centers on the $X$-axis. Some of these lines are drawn on Fig. 3.

If a circle of diameter $d$ has its center $h$ units from the horizontal axis (Fig. 2), it is easy to show (see Ref. 1) that the proper polar distance $a$ is determined from $a^{2}=h^{2}-d^{2} / 4$ and that the value $\xi_{0}$ of $\xi$ corresponding to the circle is $\xi_{0}=\cosh ^{-1} 2 h / d$. The cartesian coordinates can be expressed as follows in terms of the bipolar:

$$
x=-\frac{a \sinh \xi}{\cosh \xi-\cos \eta}, \quad y=\frac{a \sin \ddot{\eta}}{\cosh \xi-\cos \eta} .
$$

When the biharmonic equation is expressed in bipolar coordinates, it is found convenient to write it, not in terms of the usual stress function $\chi$, but in terms of $\chi / J$, where $J$ has the value

$$
J=\frac{a}{\cosh \xi-\cos \eta} ;
$$

the stresses are also expressed as derivatives of $\chi / J$. 
The bipolar solution for $\chi / J$ used by Jeffery has the general form:

$$
\begin{aligned}
\chi / J= & B \xi \cosh \xi+(-B \xi+G \cosh 2 \xi+H \sinh 2 \xi+F) \cos \eta \\
& +\left(G^{\prime} \cosh 2 \xi+H^{\prime} \sinh 2 \xi+F^{\prime}\right) \sin \eta \\
& +\sum_{k=2}^{\infty}\left\{\left[E_{k} \cosh (k+1) \xi+F_{k} \sinh (k+1) \xi+G_{k} \cosh (k-1) \xi\right.\right. \\
& \left.+H_{k} \sinh (k-1) \xi\right] \cos k \eta+\left[E_{k}^{\prime} \cosh (k+1) \xi+F_{k}^{\prime} \sinh (k+1) \xi\right. \\
& \left.\left.+G_{k}^{\prime} \cosh (k-1) \xi+H_{k}^{\prime} \sinh (k-1) \xi\right] \sin k \eta\right\},
\end{aligned}
$$

where all the $B$ 's, $E$ 's, $F$ 's, $G$ 's and $H$ 's are constants. This series will be assumed convergent and differentiable for the time being.

Here the terms independent of $\eta$ and those containing $\cos \eta$ or $\sin \eta$ are used exactly as they appear in Ref. 2 (Eq. 4.066 and paragraph 4.07), but those containing functions of multiples of $\eta$ come directly from Ref. 1 (Eq. 21), with some slight changes in nomenclature.

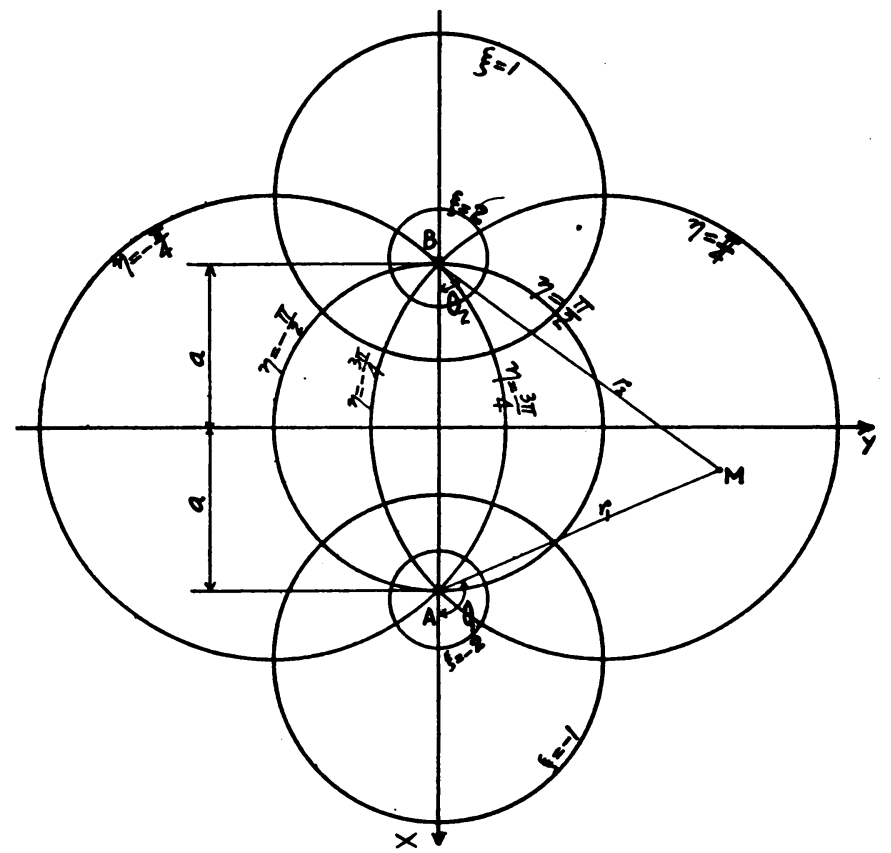

Fig. 3.

4. General procedure. As was said before, the presence of the circular discontinuity causes a modification of the Boussinesq functions $\phi_{1}$ and $\phi_{2}$ into $\Phi_{1}$, and $\Phi_{2}$ the latter having to satisfy the biharmonic equation and all boundary conditions Also $\Phi_{1}=\phi_{1}+\chi_{1}, \Phi_{2}=\phi_{2}+\chi_{2}$ where $\chi_{1}$ and $\chi_{2}$ are auxiliary stress functions of the general form (3). Now since both $\phi$ 's and both $\chi$ 's satisfy the biharmonic equation, which is linear, so do $\Phi_{1}$ and $\Phi_{2}$. As to the boundary conditions, $\phi_{1}$ and $\phi_{2}$ satisfy them along the straight boundary and for remote points. Therefore, $\chi_{1}$ and $\chi_{2}$ must be so selected that: 
(1) they give vanishing stresses for remote points $(\xi \rightarrow 0, \eta \rightarrow 0)$;

(2) they give zero normal and shearing stresses along the straight boundary;

(3) in combination with the known functions $\phi_{1}$ and $\phi_{2}$ they satisfy the boundary conditions at the circular discontinuity.

In the next paragraphs, conditions (1) and (2) will be considered first and their application will determine some of the hitherto arbitrary constants of Eq. (3). Then the function $\chi$ satisfying conditions (1) and (2) will be added to $\phi_{1}$ or $\phi_{2}$ (according to whether a normal or a tangential load is studied), yielding

$$
\Phi_{1}=\phi_{1}+\chi, \quad \Phi_{2}=\phi_{2}+\chi .
$$

Finally the remaining constants of $\chi$ will be determined in each case by the conditions at the inner boundary.

5. First and second boundary conditions. The stresses are expressed as follows in terms of bipolar coordinates:

$$
\begin{aligned}
& a \sigma_{\xi}=\left[(\cosh \xi-\cos \eta) \frac{\partial^{2}}{\partial \eta^{2}}-\sinh \xi \frac{\partial}{\partial \xi}-\sin \eta \frac{\partial}{\partial \eta}+\operatorname{ccsh} \xi\right]\left(\frac{\chi}{J}\right), \\
& a \sigma_{\eta}=\left[(\cosh \xi-\cos \eta) \frac{\partial^{2}}{\partial \xi^{2}}-\sinh \xi \frac{\partial}{\partial \xi}-\sin \eta \frac{\partial}{\partial \eta}+\cos \eta\right]\left(\frac{\chi}{J}\right), \\
& a \tau_{\xi \eta}=-(\cosh \xi-\cos \eta) \frac{\partial^{2}}{\partial \xi \partial \eta}\left(\frac{x}{J}\right) .
\end{aligned}
$$

The first condition necessitates

$$
\sigma_{\xi}=0, \sigma_{\eta}=0, \tau_{\xi \eta}=0 \text { for }(\xi, \eta) \rightarrow 0 ;
$$

and the second

$$
\sigma_{\xi}=0, \tau_{\xi \eta}=0 \text { for } \xi=0 .
$$

The first condition is seen from Eqs. (4) to be equivalent to

from which, immediately

$$
\frac{\chi}{J}=0 \text { for }(\xi, \eta) \rightarrow 0
$$

$$
G+F=0, G=-F \text { and } E_{k}+G_{k}=0, G_{k}=-E_{k} .
$$

For the second condition, $\tau_{\xi \eta}=0$ for $\xi=0$, which yields

$$
H=\frac{B}{2}, \quad H^{\prime}=0, \quad H_{k}=-\frac{k+1}{k-1} F_{k}, \quad H_{k}^{\prime}=-\frac{k+1}{k-1} F_{k}^{\prime} ;
$$

and from $\sigma_{\xi}=0$ for $\xi=0$,

$$
G_{k}^{\prime}=-E_{k}^{\prime} .
$$

Thus the stress function satisfying boundary conditions (1) and (2) assumes the form: $\frac{\chi}{J}=B \xi \cosh \xi-\left[B(\xi-\sinh \xi \cosh \xi)+2 F \sinh ^{2} \xi\right] \cos \eta+\left(G^{\prime} \cosh 2 \xi+F^{\prime}\right) \sin \eta$ 
$+\sum_{k=2}^{\infty} \frac{2}{k-1}\left\{\left[E_{k}(k-1) \sinh \xi \sinh k \xi\right.\right.$

$\left.+F_{k}(k \sinh \xi \cosh k \xi-\cosh \xi \sinh k \xi)\right] \cos k \eta$

$\left.+\left[E_{k}^{\prime}(k-1) \sinh \xi \sinh k \xi+F_{k}^{\prime}(k \sinh \xi \cosh k \xi-\cosh \xi \sinh k \xi)\right] \sin k \eta\right\}$.

6. Third boundary condition. The value of $\chi / J$ from (5) is now added to $\phi_{1}$ (for normal load) or $\phi_{2}$ (tangential load), and the remaining arbitrary constants of (5) determined by the conditions at the boundary of the hole, which are that both normal and shear stresses vanish on the periphery, i.e. $\left(\sigma_{\xi}\right)_{\xi_{0}}=0$, and $\left(\tau_{\xi_{\eta}}\right)_{\xi_{0}}=0$.

In order to coordinate the functions $\phi_{1}, \phi_{2}$, on one hand, and $\chi_{1}, \chi_{2}$, on the other, the system of axes shall be selected so that the $y=0($ or $\eta=0)$ axis passes through the center of the hole, as shown in Figs. 2 and 3. Then the concentrated force, whether normal or tangential, will act at a point $y=y_{0}$, and the stress functions $\phi_{1}$ and $\phi_{2}$ become

$$
\phi_{1}=-\frac{P}{\pi}\left(y-y_{0}\right) \tan ^{-1} \frac{y-y_{0}}{x}, \quad \phi_{2}=-\frac{P}{\pi} \tan ^{-1} \frac{y-y_{0}}{x} .
$$

Transforming this into bipolar coordinates (Eq. 2) one has

$$
\begin{aligned}
\phi_{1} & =\frac{P}{\pi} \frac{y_{0}(\cosh \xi-\cos \eta)-a \sin \eta}{\cosh \xi-\cos \eta} \tan ^{-1} \frac{y_{0}(\cosh \xi-\cos \eta)-a \sin \eta}{a \sinh \xi}, \\
\phi_{2} & =\frac{P}{\pi} \frac{a \sinh \xi}{\cosh \xi-\cos \eta} \tan ^{-1} \frac{y_{0}(\cosh \xi-\cos \eta)-a \sin \eta}{a \sinh \xi} .
\end{aligned}
$$

But, as was said before, in treating problems involving bipolar coordinates, it is easier to express stresses not in terms of the stress function itself, but in terms of the stress function divided by the quantity $J$, so that:

$$
\begin{aligned}
& \phi_{1} / J=\frac{P}{\pi a}\left[y_{0}(\cosh \xi-\cos \eta)-a \sin \eta\right] \tan ^{-1} \frac{y_{0}(\cosh \xi-\cos \eta)-a \sin \eta}{a \sinh \xi}, \\
& \phi_{2} / J=\frac{P}{\pi} \sinh \xi \tan ^{-1} \frac{y_{0}(\cosh \xi-\cos \eta)-a \sin \eta}{a \sinh \xi} .
\end{aligned}
$$

These two expressions must now be written in Fourier series in $\eta$ to be comparable with the auxiliary functions $\chi_{1}$ and $\chi_{2}$ of (5). The coefficients of these series are found by means of the usual integrations which are presented in detail in the Appendix. The results are as follows:

with

$$
\phi_{1} / J=\frac{T_{0}}{2}+\sum_{k=1}^{\infty}\left(T_{k} \cos k \eta+U_{k} \sin k \eta\right)
$$

$$
\frac{T_{0}}{2}=\frac{P}{\pi}\left\{\tan \beta \cosh \xi\left[\tan ^{-1}(\tan \beta \operatorname{coth} \xi)+\left(\frac{\pi}{2}-\beta\right) \sinh \xi\right]-e^{\xi} \frac{1+\cos \beta}{2 \cos \beta}\right\},
$$




$$
\begin{aligned}
& T_{1}=\frac{P}{\pi} \tan \beta\left[e^{\xi} \sin \beta \cosh ;-\tan ^{-1}(\tan \beta \operatorname{coth} \xi)-\left(\frac{\pi}{2}-\beta\right) \sinh \xi\right], \\
& U_{1}=\frac{P}{\pi}\left\{\frac{\tan \beta}{2}\left[1+\left(1+e^{2 \xi}\right) \cos \beta\right]-\tan ^{-1}(\tan \beta \operatorname{coth} \xi)-\left(\frac{\pi}{2}-\beta\right) \sinh \xi\right\},
\end{aligned}
$$

and for $k \geqq 2$

$$
\begin{gathered}
T_{k}=-\frac{P e^{k \xi}}{\pi}\left\{(-1)^{k} \frac{\tan \beta \sin k \beta}{k} \cosh \xi+\frac{k \sinh \xi-\cosh \xi}{k^{2}-1}\left[1+(-1)^{k} \frac{\cos k \beta}{\cos \beta}\right]\right\}, \\
U_{k}=-\frac{P e^{k \xi}}{\pi}\left\{(-1)^{k} \frac{\tan \beta \cos k \beta}{k} \cosh \xi-\frac{k \sinh \xi-\cosh \xi}{k^{2}-1}\left[\frac{\tan \beta}{k}+(-1)^{k} \frac{\sin k \beta}{\cos \beta}\right]\right\} ; \\
\phi_{2} / J=\frac{R_{0}}{2}+\sum_{k=1}^{k=\infty}\left(R_{k} \cos k \eta+S_{k} \sin k \eta\right),
\end{gathered}
$$

where

$$
\begin{aligned}
\frac{R_{0}}{2} & =\frac{P \sinh \xi}{\pi}\left[\tan ^{-1}(\tan \beta \operatorname{coth} \xi)+\left(\frac{\pi}{2}-\beta\right) \sinh \xi\right], \\
R_{k} & =-\frac{P \sinh \xi}{\pi} \frac{e^{k \xi}}{k}(-1)^{k} \sin k \beta, \\
S_{k} & =\frac{P \sinh \xi}{\pi} \frac{e^{k \xi}}{k}\left[1-(-1)^{k} \cos k \beta\right] .
\end{aligned}
$$

In these expressions $\beta=\tan ^{-1} y_{0} / a$ (Fig. 2). The above formulas refer to the case $\beta \neq 0$ (see Appendix). For the important case $\beta=0$, i.e., $y_{0}=0$

for $k \geqq 2$

$$
\frac{T_{0}}{2}=-\frac{P}{\pi} e^{\xi}, \quad T_{1}=0, \quad U_{1}=0,
$$

$$
T_{k}=-\frac{2 P}{\pi} \frac{e^{k \xi}(k \sinh \xi-\cosh \xi)}{k^{2}-1} \text { for } k \text { even, } \quad T_{k}=0 \text { for } k \text { odd, } U_{k} \doteq 0,
$$

and

$$
\frac{R_{0}}{2}=0, \quad R_{k}=0, \quad S_{k}=\frac{2 P}{\pi} \frac{e^{k \xi}}{k} \sinh \xi \text { for } k \text { odd, } \quad S_{k}=0 \text { for } k \text { even. }
$$

The following special cases will be considered in the next section on applications: (A) half-space containing a hole bounded by $\xi=\xi_{0}$ and subjected to normal load; (B) same region subjected to tangential load.

\section{APPLICATIONS}

A. Hole subject to normal load. Here

or

$$
\Phi_{1} / J=\phi_{1} / J+\chi_{1} / J,
$$


$\Phi_{1} / J=B \xi \cosh \xi+\frac{P}{\pi}\left\{\tan \beta \cosh \xi\left[\tan ^{-1}(\tan \beta \operatorname{coth} \xi)+\left(\frac{\pi}{2}-\beta\right) \sinh \xi\right]-e^{\xi} \frac{1+\cos \beta}{2 \cos \beta}\right\}$ $-\left\{B(\xi-\sinh \xi \cosh \xi)+2 F \sinh ^{2} \xi\right.$

$$
\left.-\frac{P}{\pi} \tan \beta\left[e^{\xi} \sin \beta \cosh \xi-\tan ^{-1}(\tan \beta \operatorname{coth} \xi)-\left(\frac{\pi}{2}-\beta\right) \sinh \xi\right]\right\} \cos \beta
$$

$+\left\{G^{\prime} \cosh 2 \xi+F^{\prime}+\frac{P}{\pi}\left[\frac{\tan \beta}{2}\left\{1+\left(1+e^{2 \xi}\right) \cos \beta\right\}\right.\right.$

$\left.\left.-\tan ^{-1}(\tan \beta \operatorname{coth} \xi)-\left(\frac{\pi}{2}-\beta\right) \sinh \xi\right]\right\} \sin \beta$

$+\sum_{k=2}^{\infty} \frac{2}{k-1}\left(E_{k}[k-1] \sinh \xi \sinh k \xi+F_{k}[k \sinh \xi \cosh k \xi-\cosh \xi \sinh k \xi]\right.$

$\left.-\frac{k-1}{2 \pi} P e^{k \xi}\left\{(-1)^{k} \frac{\tan \beta \sin k \beta}{k} \cosh \xi+\frac{k \sinh \xi-\cosh \xi}{k^{2}-1}\left[1+(-1)^{k} \frac{\cos k \beta}{\cos \beta}\right]\right\}\right) \cos k \eta$

$+\sum_{k=2}^{\infty} \frac{2}{k-1}\left(E_{k}^{\prime}[k-1] \sinh \xi \sinh k \xi+F_{k}^{\prime}[k \sinh \xi \cosh k \xi-\cosh \xi \sinh \xi]\right.$

$\left.-\frac{k-1}{2 \pi} P e^{k \xi}\left\{(-1)^{k} \frac{\tan \beta \cos k \beta}{k} \cosh \xi-\frac{k \sinh \xi-\cosh \xi}{k^{2}-1}\left[\frac{\tan \beta}{k}+(-1)^{k} \frac{\sin k \beta}{\cos \beta}\right]\right\}\right) \sin k \eta$.

The condition $\left(\tau_{\xi_{0}}\right)_{\xi_{0}}=-(\cosh \xi-\cos \eta) \partial^{2} / \partial \xi \partial \eta(\Phi / J)=0$ amounts to equating to zero at $\xi=\xi_{0}$ the derivative with respect to $\xi$ of each term except the one independent of $\eta$. As to $\left(\sigma_{\xi}\right)_{\xi_{0}}=0$, this can be shown to require that for $k \geqq 2$ each term be zero at $\xi=\xi_{0}$. Thus, for each term, two equations are available; and this is suffcient to find all of the remaining constants, with the exception of $F^{\prime}$ in the term in $\sin \beta$. The constant $F^{\prime}$ remains indeterminate, and can therefore be taken as equal to zero. By solving the two equations for each term, the following values are found for the constants:

$$
\begin{aligned}
& B=\frac{P}{2 \pi \sinh ^{2} \xi_{0}}\left\{e^{\xi_{0}} \tan \beta \sin \beta\left(\frac{2 \cosh ^{2} \xi_{0}}{\sinh \xi_{0}}-e^{\xi_{0}}\right)-\frac{2 \sin ^{2} \beta\left(\cosh ^{2} \xi_{0}+\frac{1}{2}\right)}{\cosh ^{2} \xi_{0}-\cos ^{2} \beta}\right. \\
& \left.-(\pi-2 \beta) \tan \beta \cosh \xi_{0}\left(\cosh ^{2} \xi_{0}-\frac{1}{2}\right)-\operatorname{coth} \xi_{0} \frac{1+\cos \beta}{\cos \beta}\right\} \text {, } \\
& F=\frac{P}{2 \pi \sinh ^{2} \xi_{0}}\left\{e^{\xi_{0}} \tan \beta \sin \beta \cosh \xi_{0}-\tan \beta \sinh \xi_{0} \cosh \xi_{0}\right. \\
& \left.\left[\frac{\sin \beta \cos \beta}{\cosh ^{2} \xi_{0}-\cos ^{2} \beta}+\left(\frac{\pi}{2}-\beta\right) \cosh \xi_{0}\right]-\frac{1+\cos \beta}{2 \cos \beta}\right\} \text {. } \\
& G^{\prime}=\frac{-P}{2 \pi \sinh 2 \xi_{0}}\left[e^{2 \xi_{0}} \sin \beta+\frac{\sin \beta \cos \beta}{\cosh ^{2} \xi_{0}-\cos ^{2} \beta}-\left(\frac{\pi}{2}-\beta\right) \cosh \xi_{0}\right], \\
& F^{\prime}=0 \text {, } \\
& E_{k}=-\frac{P}{2 \pi}\left\{(-1)^{k} \tan \beta \sin k \beta\left(k \sinh \xi_{0} \cosh \xi_{0}+\sinh ^{2} \xi_{0}-e^{k \xi_{0}} \sinh k \xi_{0}\right]\right. \\
& \left.+\left[1-(-1)^{k} \cos k \beta / \cos \beta\right] k \sinh ^{2} \xi_{0}\right\}\left[\sinh ^{2} k \xi_{0}-k^{2} \sinh ^{2} \xi_{0}\right]^{-1},
\end{aligned}
$$




$$
\begin{aligned}
& F_{k}=-\frac{P}{2 \pi}\left\{(-1)^{k}(k-1) k^{-1} \tan \beta \sin k \beta\left[k \sinh \xi_{0} \cosh \xi_{0}-e^{k \xi_{0}} \sinh k \xi_{0}\right]\right. \\
& \left.+(k+1)^{-1}\left[1+(-1)^{k} \cos k \beta / \cos \beta\right]\left[k^{2} \sinh ^{2} \xi_{0}-k \sinh \xi_{0} \cosh \xi_{0}-e^{-k \xi_{0}} \sinh k \xi_{0}\right]\right\} \\
& \quad \cdot\left[\sinh ^{2} k \xi_{0}-k^{2} \sinh ^{2} \xi_{0}\right]^{-1}, \\
& E_{k}^{\prime}=\frac{P}{2 \pi}\left\{(-1)^{k} \tan \beta \cos k \beta\left[k \sinh k \xi_{0} \cosh \xi_{0}+\sinh ^{2} \xi_{0}-e^{k \xi_{0}} \sinh k \xi_{0}\right]\right. \\
& \left.\left.\quad-\left[k^{-1} \tan \beta+(-1)^{k} \sin k \beta / \cos \beta\right] k \sinh ^{2} \xi_{0}\right]\right\}\left[\sinh ^{2} k \xi_{0}-k^{2} \sinh ^{2} \xi_{0}\right]^{-1}, \\
& F_{k}^{\prime}=\frac{P}{2 \pi}\left\{(-1)^{k}(k-1) k^{-1} \tan \beta \cos k \beta\left[k \sinh \xi_{0} \cosh \xi_{0}+e^{k \xi_{0}} \sinh k \xi_{0}\right]\right. \\
& \left.-(k+1)^{-1}\left[k^{-1} \tan \beta+(-1)^{k} \sin k \beta / \cos \beta\right]\left[k^{2} \sinh ^{2} \xi_{0}-k \sinh \xi_{0} \cosh \xi_{0}-e^{k \xi_{0}} \sinh _{k \xi_{0}}\right]\right\} \\
& \cdot\left[\sinh ^{2} k \xi_{0}-k^{2} \sinh ^{2} \xi_{0}\right]^{-1} .
\end{aligned}
$$

To test the suitability of this expansion, it is sufficient to examine the terms of the auxiliary functions (Eq. 5) for the values of the constants given above. The coefficient of $\cos k \eta$ in the general term of the latter equation is seen to consist of two parts, one multiplied by $(-1)^{k}$ and the other not. The first part forms an alternating series the general term of which tends to zero, so that the alternating series is convergent by a well-known theorem. The second part is found to converge outside the circle $\xi=\xi_{0}$ by the ratio test. The same is true for the coefficient of $\sin k \eta$. Thus the above expression for $\Phi_{1} / J$ is a uniformly convergent series in $\eta$ in the region considered.

Because of the great complexity of the expression involved, only the case $\beta=0$ will be considered in more detail. For that case

$$
\begin{aligned}
& B^{(0)}=-\frac{P}{\pi} \frac{\cosh \xi_{0}}{\sinh ^{3} \xi_{0}}, \quad F^{(0)}=-\frac{P}{2 \pi \sinh ^{2} \xi_{0}}, \quad G^{\prime(0)}=F^{\prime(0)}=0, \\
& E_{k}^{(0)}=-\frac{P}{\pi} \frac{k \sinh ^{2} \xi_{0}}{\sinh ^{2} k \xi_{0}-k^{2} \sinh ^{2} \xi_{0}} \text { for } k \text { even, } \quad E_{k}^{(0)}=0 \text { for } k \text { odd, } \\
& F_{k}^{(0)}=-\frac{P}{\pi} \frac{k^{2} \sinh ^{2} \xi_{0}-k \sinh \xi_{0} \cosh \xi_{0}-e^{k \xi_{0}} \sinh k \xi_{0}}{(k+1)\left(\sinh ^{2} k \xi_{0}-k^{2} \sinh ^{2} \xi_{0}\right)} \text { for } k \text { even, } F_{k}^{(0)}=0 \text { for } k \text { odd, } \\
& E_{k}^{(0)}=F_{k}^{\prime(0)}=0 .
\end{aligned}
$$

The stress function becomes

$$
\begin{aligned}
\Phi_{1}^{(0)} / J= & -\frac{P}{\pi} \frac{\cosh \xi_{0}}{\sinh ^{3} \xi_{0}} \xi \cosh \xi-\frac{P}{\pi} \\
& +\frac{P}{\pi}\left[\frac{\cosh \xi_{0}}{\sinh ^{3} \xi_{0}}(\xi-\sinh \xi \cosh \xi)+\frac{\sinh ^{2} \xi}{\sinh ^{2} \xi_{0}}\right] \cos \eta \\
& -\sum_{k=2}^{\infty} \frac{2 P}{\pi(k-1)}\left[\frac{k(k-1) \sinh ^{2} \xi_{0}}{\sinh ^{2} k \xi_{0}-k^{2} \sinh ^{2} \xi_{0}} \sinh \xi \sinh k \xi\right.
\end{aligned}
$$




$$
\begin{aligned}
& +\frac{k^{2} \sinh ^{2} \xi_{0}-k \sinh \xi_{0} \cosh \xi_{0}-e^{k \xi_{0}} \sinh k \xi_{0}}{(k+1)\left(\sinh ^{2} k \xi_{0}-k^{2} \sinh ^{2} \xi_{0}\right)}(k \sinh \xi \cosh k \xi-\cosh \xi \sinh k \xi) \\
& \left.+\frac{e^{k \xi}}{k+1}(k \sinh \xi-\cosh \xi)\right] \cos k \eta
\end{aligned}
$$

with the summation extending over even values of $k$ only.

The most significant stress is the hoop stress $\sigma$, at the periphery of the hole, $\xi=\xi_{0}$. Substituting the above value of $\Phi_{1} / J$ into the second of Eqs. (4), the following series is obtained:

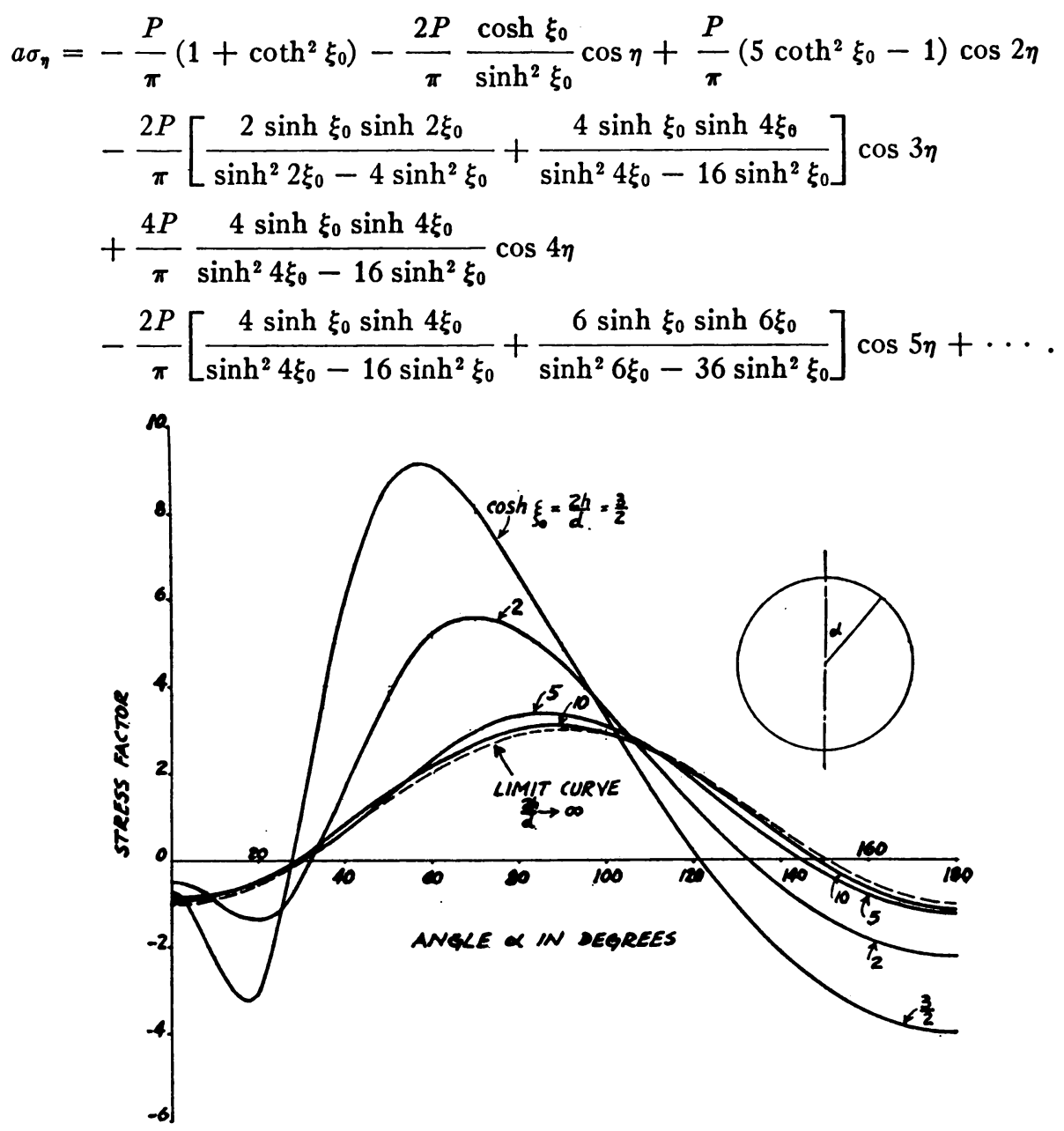

FIG. 4.

Fig. 4 is a graphical illustration of the above formula. In that figure, the "stress factor" is plotted for different values of $2 h / d=\cosh \xi_{0}$. By "stress factor" is meant the ratio of the stress $\sigma$, to the stress which would have existed under the same loading at a point corresponding to the center of the hole, if the latter had not been 
drilled. If there had been no hole, the point corresponding to its center would have been under a stress $-2 P / \pi h$ (compression) so that the stress factor is the ratio $-\sigma_{\eta} /(2 P / \pi h)$. Therefore, a positive value of the stress factor represents compression, a negative value, tension.

It is seen from the figure that for each curve there exists a tension directly under the load $(\alpha=0)$, which becomes a compression as $\alpha$ is increased, reaches a maximum, then decreases, and becomes tension again when $\alpha$ approaches $180^{\circ}$. For low values of $\cosh \xi_{0}$, i.e., of the depth-to-diameter ratio, there exists a secondary maximum of tension in the neighborhood of $\alpha=20^{\circ}$.

As cosh $\xi_{0}$ increases (as the hole gets deeper and deeper), the stress factor curves tend towards the "limit curve," which is simply the graph of $1-2 \cos 2 \alpha$. The latter expression (Ref. 4, p. 77, second of Eqs. (58)) is obtained by assuming the hole to be in a field of uniform compression, equal to the compression $-2 P / \pi h$ at the center of the hole.

B. Hole subjected to tangential load. Now the total stress function has the form

$$
\Phi_{2} / J=\varphi_{2} / J+\chi_{2} / J,
$$

where $\Phi_{2}$ is the total stress function, $\phi_{2}$ is given by (1b) and $\chi_{2}$ is of the general form (5). The heretofore arbitrary constants are determined by the conditions at the inner boundary, which are the same as in the preceding case. The remaining constants are found to be:

$$
\begin{aligned}
B & =\frac{P}{2 \pi}\left[\frac{\sin \beta}{\sinh ^{2} \xi_{0}}+\frac{\sin 2 \beta \operatorname{coth} \xi_{0}}{\cosh ^{2} \xi_{0}-\cos ^{2} \beta}-(\pi-2 \beta) \frac{\cosh ^{2} \xi_{0}}{\sinh \xi_{0}}\right], \\
F & =\frac{P}{2 \pi}\left[\frac{e^{\xi_{0}} \sin \beta}{\sinh \xi_{0}}+\frac{\sin \beta \cos \beta}{\cosh ^{2} \xi_{0}-\cos ^{2} \beta}-\left(\frac{\pi}{2}-\beta\right) \cosh \xi_{0}\right], \\
G^{\prime} & =-\frac{P}{2 \pi} \frac{e^{2 \xi_{0}}}{\sinh 2 \xi_{0}}(1+\cos \beta), \\
F^{\prime} & =0, \\
E_{k} & =-\frac{P}{2 \pi}(-1)^{k} \frac{\sin k \beta}{k} \frac{k^{2} \sinh ^{2} \xi_{0}+k \sinh \xi_{0} \cosh \xi_{0}-e^{k \xi_{0} \sinh k \xi_{0}}}{\sinh ^{2} k \xi_{0}-k^{2} \sinh ^{2} \xi_{0}}, \\
F_{k} & =-\frac{P}{2 \pi}(-1)^{k}(k-1) \sin k \beta \frac{\sinh ^{2} \xi_{0}}{\sinh ^{2} k \xi_{0}-k^{2} \sinh ^{2} \xi_{0}}, \\
E_{k}^{\prime} & =\frac{P}{2 \pi} \frac{\left[1-(-1)^{k} \cos k \beta\right]}{k} \frac{k^{2} \sinh ^{2} \xi_{0}+k \sinh ^{2} \xi_{0} \cosh _{0}-e^{k \xi_{0} \sinh k \xi_{0}}}{\sinh ^{2} k \xi_{0}-k^{2} \sinh ^{2} \xi_{0}} \\
F_{k}^{\prime} & =\frac{P}{2 \pi}(k-1)\left[1-(-1)^{k} \cos k \beta\right] \frac{\sinh ^{2} \xi_{0}}{\sinh ^{2} k \xi_{0}-k^{2} \sinh ^{2} \xi_{0}} .
\end{aligned}
$$

The resulting Fourier series can be shown to converge as in the previous case.

C. Conclusion. In the above paragraphs, a method was presented for computing the distortion of the original Boussinesq field when a hole is introduced. Other interesting results can be derived by simple means; thus, by superposing on the above stress functions $\Phi_{1}$ or $\Phi_{2}$ one of the solutions presented in Refs. 1 and 2, it is possible 
to obtain the stress system for a Boussinesq field containing a hole, the periphery of which is subjected to a uniform pressure. Another extension of the above method, on which the writer is working at present, can be used to solve the case of a Boussinesq field containing a rigid disc.

\section{APPENDIX}

The decomposition of the Boussinesq stress function into a Fourier series in $\eta$. We shall begin by decomposing the shear stress function

$$
\frac{\phi_{2}}{J}=\frac{P}{\pi} \sinh \xi \tan ^{-1} \frac{y_{0}(\cosh \xi-\cos \eta)-a \sin \eta}{a \sinh \xi} .
$$

The different Fourier coefficients are given by

$$
R_{k}+i S_{k}=\frac{1}{\pi} \int_{0}^{2 \pi} \frac{\phi_{2}}{J} e^{i k \eta} d \eta .
$$

This can be simplified by introducing the angle $\beta=\tan ^{-1} y_{0} / a$.

$$
\begin{aligned}
\frac{\phi_{2}}{J} & =\frac{P}{\pi} \sinh \xi \tan ^{-1} \frac{y_{0}(\cosh \xi-\cos \eta)-a \sin \eta}{a \sinh \xi} \\
& =\frac{P}{\pi} \sinh \xi \tan ^{-1} \frac{\sin \beta \cosh \xi-\sin (\eta+\beta)}{\cos \beta \sinh \xi}
\end{aligned}
$$

Let also $\sin \beta \cosh \xi=p, \cos \beta \sinh \xi=q, \eta+\beta=\psi$. Then

$$
\begin{aligned}
R_{k}+i S_{k} & =\frac{1}{\pi} \int_{0}^{2 \pi} \frac{P}{\pi} \sinh \xi \tan ^{-1} \frac{p-\sin \psi}{q} e^{i k \eta} d \eta \\
& =\frac{P \sinh \xi e^{-i k \beta}}{\pi^{2}} \int_{0}^{2 \pi} \tan ^{-1} \frac{p-\sin \psi}{q} e^{i k \psi} d \psi=\frac{P \sinh \xi e^{-i k \beta}}{\pi^{2}} I_{k} .
\end{aligned}
$$

Here the limits of integration need not be changed, since the integrand is a periodic function of period $2 \pi$. To evaluate $I_{k}$, use is made of integration by parts, with

Then

$$
u=\tan ^{-1} \frac{p-\sin \psi}{q}, \quad d v=e^{i k \psi} d \psi .
$$

$$
d u=-\frac{q \cos \psi d \psi}{(p-\sin \psi)^{2}+q^{2}}, \quad v=-\frac{i}{k} e^{i k \psi} \quad(k \neq 0)
$$

and

$$
I_{k}=\left.u \vartheta\right|_{0} ^{2 \pi}-\int_{0}^{2 \pi} v d u .
$$

But, since both $u$ and $v$ are periodic, their product evaluated over the period $2 \pi$ is zero; then

$$
I_{k}=-\int_{0}^{2 \pi} v d u=-\frac{i q}{k} \int_{0}^{2 \pi} \frac{\cos \psi d \psi}{(p-\sin \psi)^{2}+q^{2}} e^{i k \psi} .
$$


Replacing the sine and cosine by their exponential equivalents and transforming, one has

$$
I_{k}=\frac{2 q}{k} \int_{0}^{2 \pi} e^{i k \psi} \frac{\left(e^{2 i \psi}+1\right) d\left(e^{i \psi}\right)}{\left(e^{2 i \psi}-2 i p e^{i \psi}-1\right)^{2}-4 q^{2} e^{2 i \psi}} .
$$

This is easily seen to be a rational function of $e^{i \psi}$, the denominator of which, the difference of two squares, can be decomposed into two quadratic factors with relatively simple roots, so that the transformation by partial fractions can be used to obtain the following result:

$$
I_{k}=\frac{1}{2 k} \int_{0}^{2 \pi} e^{i k \psi}\left(\frac{1}{e^{i \psi}-e^{\xi} e^{i \beta}}-\frac{1}{e^{i \psi}-e^{-\xi} e^{i \beta}}-\frac{1}{e^{i \psi}+e^{\xi} e^{-i \beta}}+\frac{1}{e^{i \psi}+e^{-\xi} e^{-i \beta}}\right) d\left(e^{i \psi}\right) .
$$

Thus the integral breaks down into four integrals of the form

$$
\int_{0}^{2 \pi} \frac{t^{k}}{t-c} d t
$$

where $t=e^{i \downarrow}$, and $c$ is a complex constant of the form $\pm e^{ \pm \xi \pm i \beta}$. Now if the indicated division of $t^{k}$ by $t-c$ is performed, a quotient which is a polynomial in $t$ and $a$ remainder $c^{k}$ result. The polynomial is integrated into another polynomial in $t=e^{i \downarrow}$, and the value of this second polynomial between the limits 0 and $2 \pi$ is zero because of the periodicity of $e^{i \psi}$. Thus the remaining terms are of the type

namely,

$$
\int_{0}^{2 \pi} \frac{c^{k} d t}{t-c}=\int_{0}^{2 \pi} \frac{c^{k}}{e^{i \psi}-c} d\left(e^{i \psi}\right)
$$

$$
I_{k}=\frac{1}{2 k} \int_{0}^{2 \pi}\left[\frac{\left(e^{\xi} e^{i \beta}\right)^{k}}{e^{i \psi}-e^{\xi} e^{i \beta}}-\frac{\left(e^{-\xi} e^{i \beta}\right)^{k}}{e^{i \psi}-e^{-\xi} e^{i \beta}}-\frac{\left(-e^{\xi} e^{-i \beta}\right)^{k}}{e^{i \psi}+e^{\xi} e^{-i \beta}}+\frac{\left(-e^{-\xi} e^{-i \beta}\right)^{k}}{e^{i \psi}+e^{-\xi} e^{-i \beta}}\right] d\left(e^{i \psi}\right) .
$$

The value of the resulting terms can be obtained more easily by considering the corresponding complex function of $\zeta=\psi+i \omega$. $^{*}$

$$
c^{k} \int_{c} \frac{d\left(e^{i \zeta}\right)}{e^{i \zeta}-c}=i c^{k} \int_{c} \frac{e^{i \zeta} d \zeta}{e^{i \zeta}-c}
$$

along the contour shown on Fig. 5. It is well known from the theory of the complex variable that the value of the above contour integral is zero if the pole of the integrand falls outside that contour, and is equal to $2 \pi i \times i c^{k} \times$ Res., where Res. is the residue of the integrand, if the pole lies inside the contour.

Performing the integration around the contour, we obtain the following:

(1) along the real axis $-\pi \leqq \psi \leqq \pi$, the complex integral reduces to the real integral to be evaluated (limits $-\pi$ and $\pi$ are equivalent to 0 and $2 \pi$ );

(2) the two integrals along the vertical paths cancel each other;

(3) the integral along $\omega=\Lambda$ has a zero limit for $\Lambda \rightarrow \infty$.

Therefore,

\footnotetext{
* This treatment was indicated to the writer by his friend and colleague, Dr. H. G. Baerwald.
} 


$$
\int_{0}^{2 \pi} \frac{c^{k}}{e^{i \psi}-c} d\left(e^{i \psi}\right)=\left\{\begin{array}{l}
0 \text { if the pole lies outside the strip } \\
\omega \geqq 0-\pi \leqq \psi \leqq \pi \\
2 \pi i \times i c^{k} \times \text { Res. if it lies inside that strip. }
\end{array}\right.
$$

The pole occurs at $e^{i \xi}-c=0$, or $\zeta=-i \log c ; c$ is of the form

$$
c=e^{ \pm \xi} e^{i \beta} \text { or } c=-e^{ \pm \xi} e^{-i \beta}=e^{ \pm \xi} e^{i(r-\beta)} \text {, }
$$

so that $\log c= \pm \xi+i \beta$ or $\log c= \pm \xi+i(\pi-\beta)$ and $\zeta=\beta \mp i \xi$ or $\zeta=\pi-\beta \mp i \xi$.

But the region $x>0$ corresponds to $\xi<0$, as can be seen from (2), and also $0<\beta<\pi / 2$, so that the integrals whose pole has an imaginary part of the form $+i \xi$, namely the second and fourth of (A), have the value zero. The poles of the first and third, on the contrary, fall inside the region of integration, so that their values are

$$
2 \pi i \times i c^{k} \times \text { Res. }
$$

It remains to evaluate the residue. This is found to be $-i$ by methods explained in texts on the complex variable (Ref. 5). Thus the required integrals become

Thus,

$$
2 \pi i \times i c^{k} \times(-i)=2 \pi i c^{k} \text {. }
$$

and

$$
I_{k}=\frac{1}{2 k} 2 \pi i\left[\left(e^{k} e^{i \phi}\right)^{k}-\left(-e^{k} e^{-i}\right)^{k}\right]
$$

$$
R_{k}+i S_{k}=\frac{P i}{\pi} \sinh \xi \frac{e^{k \xi}}{k}\left[1-e^{i(\pi-\beta) k}\right]
$$

Therefore

$$
\begin{aligned}
& R_{k}=-(-1)^{k} \frac{P}{\pi} \sinh \xi \sin k \beta \frac{e^{k \xi}}{k}, \\
& S_{k}=\frac{P}{\pi} \sinh \xi \frac{e^{k \xi}}{k}\left[1-(-1)^{k} \cos k \beta\right] .
\end{aligned}
$$

When $\beta=0$, the poles shown on Fig. 5 have real parts 0 and $\pi$, respectively. In other words, one of the poles is on the contour itself. Besides, due to the periodicity of the integrand, a third pole appears with a real part equal to $-\pi$. This latter pole has, in general, a real part $-\pi-\beta$, and is identical with the pole at $\pi-\beta$. Thus, there are three poles in all, one wholly within the contour and two others, with equal residues, on the contour itself. Now it is easy to see that each of the latter contributes half its residue to the value of the integral, and since these residues are equal, the situation remains the same as if there were only two poles, both entirely within the contour, so that the case $\beta=0$ is not essentially different from $\beta \neq 0$, and it is sufficient to set $\beta=0$ in the above formulas for $R_{k}$ and $S_{k}$. Thus

$$
R_{k}^{(0)}=0, \quad S_{k}^{(0)}=\frac{2 P}{\pi} \frac{e^{k \xi}}{k} \sinh \xi \quad \text { for } k \text { odd, } \quad S_{k}^{(0)}=0 \text { for } k \text { even. }
$$

Case $k=0$. For this case, the procedure is exactly the same up to the integration by parts. There, while $u$ remains as before, $d v=d \psi, v=\psi$, so that $I_{k}$ becomes 


$$
I_{0}=\left.\psi \tan ^{-1} \frac{p-\sin \psi}{q}\right|_{0} ^{2 \pi}+\int_{0}^{2 \pi} \frac{q \psi \cos \psi d \psi}{(p-\sin \psi)^{2}+q^{2}}=2 \pi \tan ^{-1} p / q+q J_{0} .
$$

To evaluate $J_{0}$, the same method as before is used, exponentials being introduced in place of the trigonometric functions:

$$
J_{0}=2 i \int_{0}^{2 \pi} \frac{\psi\left(e^{2 i \psi}+1\right) d\left(e^{i \downarrow}\right)}{\left(e^{2 i \psi}-2 i p e^{i \downarrow}-1\right)^{2}-4 q^{2} e^{2 i \psi}} .
$$

This can again be transformed into partial fractions:

$$
J_{0}=\frac{i}{2 \cos \beta} \int_{0}^{2 \pi} \psi\left[\frac{1}{e^{i \psi}-e^{\xi} e^{i \beta}}-\frac{1}{e^{i \psi}-e^{-\xi} e^{i \beta}}-\frac{1}{e^{i \psi}+e^{\xi} e^{-i \beta}}+\frac{1}{e^{i \psi}+e^{-\xi} e^{-i \beta}}\right] d\left(e^{i \psi}\right) .
$$

Here we are dealing with integrals of the type

$$
\int_{0}^{2 \pi} \frac{\psi d\left(e^{i \psi}\right)}{e^{i \psi}-c}=i \int_{0}^{2 \pi} \frac{\psi e^{i \psi} d \psi}{e^{i \psi}-c}
$$

these can be treated, as before, by introducing the complex variable and integrating around the contour of Fig. 5. Since the denominator of the integrand is the same as before, all that was said about the poles of the partial integrals making up $I_{k}$ remains true. Therefore, the second and fourth terms in the expression for $J_{0}$ contribute nothing, and the first and third are each equal to $2 \pi i$ times the residue times constants. The residues,

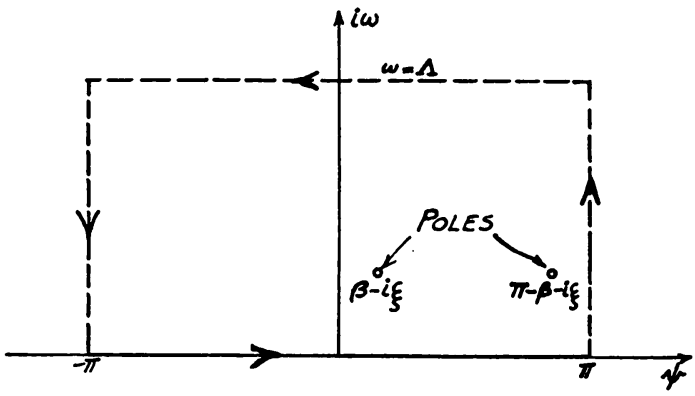

FIG. 5. however, have here the value $-\log c$, so that

$$
J_{0}=\frac{i}{2 \cos \beta} i 2 \pi i[-\xi-i \beta+\xi+i(\pi-\beta)]=\frac{\pi}{\cos \beta}(\pi-2 \beta),
$$

and the imaginary term vanishes, as could be expected. Then

$$
I_{0}=2 \pi \tan ^{-1}(\tan \beta \operatorname{coth} \xi)+\pi(\pi-2 \beta) \sinh \xi
$$

and

$$
R_{0}=\frac{P \sinh \xi}{\pi}\left[2 \tan ^{-1}(\tan \beta \operatorname{coth} \xi)+(\pi-2 \beta) \sinh \xi\right] .
$$

Half of this expression is the first term of the Fourier series:

$$
\frac{R_{0}}{2}=\frac{P \sinh \xi}{\pi}\left[\tan ^{-1}(\tan \beta \operatorname{coth} \xi)+\left(\frac{\pi}{2}-\beta\right) \sinh \xi\right] .
$$


For $\beta=0$, there are again three poles, one on the imaginary axis and the two others with real parts $\pm \pi$. This location is, as in the general case $k \neq 0$, due to the periodicity of the denominator of the integrand. However, here the integrand as a whole is not periodic, so that the residues at the two poles on the contour are not equal, and the situation is not the same as for $\beta \neq 0$. The detailed computations show that $R_{0}=0$.

Series for $\phi_{1} / J$. Since the ratio

$$
\phi_{1} / J: \phi_{2} / J=\phi_{1} / \phi_{2}=\frac{p-\sin (\eta+\beta)}{q}
$$

is a simple trigonometric expression, the series for $\phi_{1} / J$ can be obtained from that for $\phi_{2} / J$ by term-by-term multiplication.

\section{REFERENCES}

1. G. B. JefFery, Plane stress and plane strain in bipolar coordinates, Philos. Trans. of the Royal Society, London, A 221, 265 (1921).

2. E. G. Coker and L. N. G. Filon, Photoelasticity, Cambridge University Press, 1931, p. 306.

3. R. D. Mindin, Stress distribution around a tunnel, Trans. of the A.S.C.E., 105, 1117 (1940).

4. S. Timoshenko, Theory of elasticity, McGraw-Hill, 1934.

5. N. W. McLachlan, Complex variable and operational calculus, Macmillan, 1942, p. 43. 